\title{
El canto en las músicas populares: reflexiones en torno a su abordaje y enseñanza
}

Daniel Machuca Téllez

Laboratorio para el estudio de la experiencia musical, Facultad de Bellas Artes, Universidad Nacional de La Plata, La Plata, Argentina

danirichi90@gmail.com

Mónica Valles

Laboratorio para el estudio de la experiencia musical, Facultad de Bellas Artes, Universidad Nacional de La Plata, La Plata, Argentina

monicavalles146@gmail.com

\section{Resumen}

A lo largo de la historia el canto ha tenido un lugar fundamental en la práctica musical por lo que se constituyó como una habilidad a desarrollarse en los espacios de formación del músico profesional. Los caminos para llegar a esto han sido muy diversos. Sin embargo, un modo de producción vocal académico, basado en el estilo y la estética lírico/coral, aparece repetidamente para consolidarse como prototipo en el abordaje de las distintas formas de canto, sin tomar en consideración el contexto, la heterogeneidad e identidad propia de cada estilo. Este artículo pretende reflexionar acerca del devenir en la enseñanza del canto y, en particular, su relación con el abordaje de las músicas populares.

Palabras clave: voz, canto, enseñanza, músicas populares, tradición

\section{Singing in Popular Music: Reflections on its Approach and Teaching}

\begin{abstract}
Throughout history, singing has had a fundamental place in musical practice, which is why it became a skill to be developed in the professional musician's training spaces. The paths to achieve this have been very
\end{abstract}


diverse. However, an academic vocal production mode, based on lyrical/ choral style and aesthetics, appears repeatedly to consolidate itself as a prototype in the approach to different forms of singing, without taking into account the context, heterogeneity and identity of each style. This article tries to reflect on the future of song teaching and, in particular, its relationship with the approach of popular music.

Keywords: Voice, singing, teaching, popular music, tradition

\section{O canto na música popular: reflexões sobre a sua abordagem e ensino}

\section{Resumo}

Ao longo da história, o canto ocupou um lugar fundamental na prática musical, razão pela qual se tornou uma habilidade a ser desenvolvida nos espaços de treinamento dos músicos profissionais. Os caminhos para conseguir isso foram muito diversos. No entanto, um modo de produção vocal acadêmico, baseado no estilo lírico/coral e na estética, parece consolidarse repetidamente como um protótipo na abordagem de diferentes formas de canto, sem levar em conta o contexto, a heterogeneidade e a identidade de cada estilo. Este artigo tem como objetivo refletir sobre o futuro do ensino de canções e, em particular, a sua relação com a abordagem da música popular.

Palavras-chave: voz, canto, ensino, música popular, tradição

Existen variadas formas en las cuales el tratamiento de la voz en el canto puede ser abordado y entendido. A través del tiempo se ha intentado conocer y comprender su funcionamiento y los modos que permiten desarrollarla para dicha actividad, siendo aún, un campo en discusión.

La historia, las investigaciones y los avances respecto al tema, suelen contextualizarse en un marco de tradición principalmente centroeuropea ligada a la estética del canto lírico/coral. El espacio en que se desarrolla dicha práctica, atiende particularmente a detalles de la emisión muy puntuales de su estilo y estética. Cuestiones como la homogeneidad del timbre, la perfecta impostación, el tipo de respiración, la precisión fonética, la intensidad elevada entre otros, son aspectos fundamentales que configuran su producción (Cobeta, Núñez y Fernández, 2013). Es justamente en esta historia y tradición del modo de producción vocal del canto lírico/ 
coral, que la enseñanza se ha generalizado y circunscripto a sus prácticas, conceptos e imaginarios relacionados con lo innato, el talento y el virtuosismo, en la gran mayoría de músicas. De este modelo provienen la mayor parte de los métodos utilizados en occidente para el desarrollo de habilidades de ejecución y el abordaje de repertorio, aún en otros espacios de práctica músico-vocales muy diferentes. Tal es el caso de las músicas populares, que poseen características propias en sus modos de desarrollo y producción vocal que difieren al menos en lo que concierne a características estéticas y metodológicas y que implican cuestiones contextuales, prácticas e identitarias. Ejemplo de ello son las voces del growl utilizado en el estilo musical death metal, donde el grito, rasguido y gutural, son característicos de su identidad musical, también utilizadas en el rock, hardcore, punk, entre otros. Así mismo, la fuerte nasalización en la murga o el candombe, un poco menos en la salsa y la cumbia argentina, la voz entubada y llevada muy atrás de la cavidad faríngea en la cumbia y algunos estilos del folclore colombiano y mexicano, el aireado de la balada y el pop, etc., pueden ayudarnos a identificar las significativas diferencias con el paradigma tradicional. En consecuencia, se genera una tensión al abordar -por ejemplo- la enseñanza del death metal bajo un paradigma que no le es cercano.

En relación con lo anterior, nos proponemos en este trabajo reflexionar en torno a las complejidades que emergen en el abordaje y enseñanza de la voz cantada en las denominadas músicas populares. En primer lugar, haremos algunas consideraciones acerca del modelo tradicional para luego, recorrer las metodologías que subyacen a este modelo y con las que intentan el abordaje de músicas populares. A su vez, expondremos una propuesta metodológica que, consideramos, aporta y enriquece el espacio de enseñanza con modos de producción vocal próximos a las emisiones de músicas populares, así como sus implicancias pedagógicas. Finalmente, realizaremos algunas consideraciones a modo de conclusión.

\section{El peso de la tradición}

Es difícil pensar el canto y su pedagogía en Latinoamérica sin la influencia de la tradición académica centroeuropea. En el periodo colonial, comprendido entre los siglos XVI al XIX, las técnicas, estilos y estéticas europeas se trasladaron en las misiones colonizadoras, acaparando la mayor parte del territorio americano y logrando que las comunidades originarias adoptaran con bastante rapidez las formas e instrumentos que acompañaban estas músicas. Si bien los modelos centroeuropeos incorporaron 
características de la práctica nativa que le confirieron, de alguna manera, cierto carácter identitario, aun así, compositores que no traspasaron las fronteras externas de América, dejaron obras con una impronta europea comparable con el estilo barroco italiano (Barriga, 2006).

En este sentido, el tratamiento de la voz en la práctica musical latinoamericana se circunscribió al modelo colonial. De ahí que, las principales consideraciones sobre la voz y su posterior desarrollo, hayan tenido como base referencial el contexto lírico/coral y operístico. No obstante, debemos comprender el entramado de la voz en este contexto para situarnos en el porqué de su generalidad en la práctica educativa.

La voz como un instrumento musical de gran sonoridad y proyección se instauró con la proliferación de la armonía contrapuntística y la polifonía. El avance en términos técnicos del canto lírico se inicia en el siglo XVI y posteriormente se ampliaría en el siglo XVII con el florecimiento de la ópera, la oratoria, la cantata y el aria (Stein, 2000). Estas, adquirieron mayores características en función del desarrollo estético del estilo lírico hasta el siglo XX. Sin embargo, la concepción del canto como habilidad/ dominio técnico de la voz, viene del periodo romántico o la ópera del siglo XVIII, en la cual, la voz bella y el bel canto se desarrollarían como idea de culto. Fue de hecho, la inclusión del canto de exhibición y el virtuosismo de los castrati ${ }^{1}$ en la ópera cómica napolitana, lo que marcó un hito en lo que respecta a las posibilidades de la voz. Con la posterior desaparición de los castrati, que permitió articular una pedagogía en el canto, emerge un desarrollo metodológico constante (Perelló, Caballé y Guitart, 1982; Cobeta, Núñez y Fernández, 2013). A partir de ello, la fundación en 1795 del Conservatorio Nacional de París cimentó las bases de formación musical profesional y el crecimiento de métodos de enseñanza institucional, que encontramos aún en diversos ámbitos de enseñanza en el mundo y que consolidan el modelo conservatorio (Kingsbury, 1988; Musumeci, 2002; López y Vargas, 2010; Hemsy de Gainza, 2002).

En ese devenir, la enseñanza del canto tuvo un desarrollo que derivo en varias escuelas (italiana, alemana, francesa y española) que fueron perfilando el camino metodológico de la práctica. Aunque cada una se constituyó con atributos particulares, todas adscribieron a máximas de la estética propia de las músicas académicas en las que el virtuosismo e

\footnotetext{
${ }^{1}$ Castrati es el plural del italiano castrato, que se refiere a los miembros de una agrupación masculina, castrados para mantener la voz aguda infantil y así tener una carrera musical. Castrato, evirato, capón, caponcillos, capado, míseros, tiples, falsetistas, dessus mués, sopranista, kastrat, son algunas de las formas idiomáticas utilizadas para nombrar a los castrati (Augustin, 2012).
} 
ideales de pureza y claridad como parámetros de belleza en la emisión sonora, se constituyeron como un acervo hegemónico en la enseñanza. Los aportes de estas escuelas se vincularon principalmente a la música vocal de tradición europea y el paradigma asociado a las bellas artes, dejando por fuera las prácticas de músicas populares de espacios rurales y urbanos que acontecían en paralelo tanto en América como en Europa. Tales escuelas vinculadas principalmente con el canto lírico/coral, establecieron un modelo a seguir bajo el supuesto de ser adecuado y eficaz incluso en el abordaje de la voz en las músicas populares; desde esta posición, el canto en general se interpretaría con base en dicho modelo.

Estos acontecimientos en la historia han contribuido al distanciamiento de los espacios de práctica -académico y popular/rural/urbano--, muchas veces marcados por las posibilidades y características propias de cada propuesta. Las prácticas del canto lírico implican una alta exigencia desde el punto de vista técnico vocal y escénico y la necesidad de tener un aparato vocal equilibrado y en las mejores condiciones como aspecto esencial en la producción de la voz. Así, el virtuosismo es uno de los aspectos más relevantes en estos modelos en los que otras cuestiones, tales como las textuales, las semánticas o la precisión fonética, quedan en un segundo plano y los cantantes pueden transmitir significado o ejecutar matices no explícitos en la partitura; una cuestión que marcaría un contraste con los cantantes de músicas populares (Lopes, Basso y de Andrade, 2015; Sánchez, Guimarães y de Andrade, 2017).

Ahora bien, el desarrollo de las técnicas vocales de este modelo ha estado orientado a la resolución de problemas vinculados con la altura y duración del sonido, la dinámica, la tímbrica, la interpretación, etc.; estas se han convertido en el prototipo para abordar músicas de toda procedencia.

Si bien desde hace algunos años, distintos profesionales de la música se han interesado por la voz en estilos de la música popular, no deja de estar presente la perspectiva del modelo tradicional. Referentes como Stephen Zegree (2002) o Kirby Shaw (1987) entre otros, han hecho diversos aportes al tema. Ambos arreglistas y músicos de jazz vocal han sido pioneros en ofrecer recursos para el estilo y conjunto de jazz vocal a los directores de coros y ensambles. Si bien estos avances constituyen un gran aporte para conocer las características del estilo, las metodologías que proponen están basadas en parámetros del modelo tradicional, en particular del tratamiento de la voz o la producción de sonidos, mezcla y equilibrio del coro tradicional de Europa occidental. Por su parte, Anne Peckham (2000 y 2006) desarrolla un conjunto de ejercicios de vocalización para 
estilos como jazz, pop y rock, a la vez que incluye una breve introducción a cuestiones de postura y cuidado de la voz. Más allá de la variedad para el estudio del canto, su propuesta se basa en el tratamiento tradicional de la técnica vocal. Así mismo, el cantante Norman Spivey (2005 y 2008), ha hecho contribuciones a la enseñanza y producción de la voz en el teatro musical, aunque basadas en su experiencia como cantante lírico y desde la perspectiva académica que nutrió su formación.

\section{Metodologías subyacentes}

La enseñanza del canto desde la salida de los castrati de la escena musical ha tomado diferentes caminos en torno a la concepción y desarrollo de la voz, ampliando la mirada a un nivel ontológico y focalizando las diferencias con la voz hablada. Esto ha permitido, desde finales del siglo XIX, amplificar los conocimientos de la producción del sonido vocal y acentuar en la enseñanza algunos atributos más relevantes según la perspectiva de abordaje. De este modo, podemos encontrar -además del modelo tradicional- dos modelos con sus metodologías generales que abordan la voz desde concepciones e ideologías diferenciadas: (i) el modelo de salud e higiene vocal (Machuca, Valles y Pérez, 2019) y (ii) el modelo de experiencia corporal.

El primero es un modelo similar al tradicional, pudiéndose considerar como una bifurcación de este. Surge a mediados del siglo XIX cuando se inicia la exploración de las cuerdas vocales a partir de una metodología de observación y descripción; esto se alcanza gracias a la utilización de un espejo de dentista introducido en la cavidad bucal (Guerrero, 2015). El cantante y maestro Manuel Patricio Rodríguez Sitches, conocido como Manuel García, escribiría a partir de sus observaciones, el Tratado completo del arte del canto de 1840. En el mismo detalla algunas características del funcionamiento del aparato vocal, las cuerdas vocales, los aspectos relevantes en la comprensión de las voces masculinas, femeninas e infantiles, así como la descripción de una serie de ejercicios de entrenamiento vocal con su respectivo proceder. Por supuesto, su libro se enmarca en un contexto vocal operístico dado que García sostenía una activa carrera musical como barítono.

Si bien, García no define en profundidad el funcionamiento saludable de las cuerdas vocales debido a las limitantes tecnológicas del momento, se considera como un disparador para el inicio de un fuerte movimiento de investigación sobre el funcionamiento de la voz. De allí, se derivan hasta hoy, investigaciones de suma importancia que realizaron un gran aporte al 
conocimiento del aparato vocal y su desenvolvimiento como instrumento en la comunicación y el canto. Así mismo, los descubrimientos impactaron en disciplinas como la medicina, foniatría, psicología, logopedia y toda otra disciplina en la cual se veía involucrada la voz. Músicos e investigadores como Raoul Husson (1965); Johan Sundberg (1979); François Le Huche y André Allali (2004) e Ingo Titze (2010); entre otros, han profundizado en la voz cantada, gracias al interés por comprender sus experiencias como cantantes y en muchos casos como maestros de canto.

Esta información se convirtió en un insumo para repensar las metodologías pasadas que se utilizaban en la enseñanza del canto. Esto puede verse materializado en la aparición de innumerable cantidad de literatura referida a la voz, así como de maestros que propusieron alternativas con base en estos marcos conceptuales y teóricos. Es importante mencionar que, varios estudios realizados posteriormente sobre los modos de producción vocal en el canto lírico/coral, reafirman la idea de calidad, eficacia y superioridad de este tipo de canto en contraste con las músicas populares y folclóricas. El fundamento y justificación de estos estudios y resultados, se encuentra relacionado con la configuración fisiológica del aparato vocal en el cual, se considera que toda disposición en la que se vea involucrado algún tipo de sobreesfuerzo muscular o en órganos próximos al mismo, constituye un camino propicio para el deterioro y formación de patologías vocales (nódulos, pólipos, hiatus cordal, etc.). En este sentido, el canto también llamado clásico o culto, es considerado la base para cualquier modo de producción vocal, configurando así una estrecha relación con el modelo de salud vocal.

Profundizando un poco sobre este modelo, algunas características que nos permiten identificarlo en un marco de enseñanza podrían estar relacionadas con la concepción del sonido emitido. La producción de este debe suponer el cuidado total, claridad/limpieza, homogeneidad entre un sonido y otro, así como la constitución de un sonido robusto y lleno. El canto con este tipo de resultante sonora es una habilidad que puede ser desarrollada a partir de una técnica apropiada que cultive tales atributos, usando como ejemplo prototípico el modo de producción vocal de tradición clásica. El cantante será entonces, quien domine la técnica de tal manera que produzca el sonido antes mencionado y que, ante un análisis laringoscópico, demuestre que su laringe se encuentra totalmente sana. Quien se dedica a la enseñanza del canto en este caso, conoce las investigaciones relacionadas al cuidado de la voz y las aplica cuidadosamente acorde a ejercitaciones que conoce y/o plantea que puedan ser adecuadas para su trabajo (Perelló et al., 1982). No obstante, en la gran mayoría de 
casos, el proceso metodológico suele ser el mismo que en el modelo tradicional. Movimientos ascendentes y descendentes por grado conjunto o intervalos que, con la articulación de vocales y silabas, van construyendo ese instrumento de emisión que servirá para el canto. Durante ese proceso de trabajo con el alumno, se observa y escucha atentamente su producción sonora, así mismo, en una suerte de retroalimentación, el dialogo entre lo que escucha el profesor y lo que siente el alumno -en términos fisiológicos-, es una constante de cada encuentro. Esto suele estar acompañado de un chequeo médico rutinario del aparato vocal que permite al profesor constatar y tener mayor certeza del camino que decidió adoptar con determinado alumno.

Independientemente de las exigencias que se presumen del canto clásico, una voz desarrollada bajo este modelo de salud e higiene, no debiera presentar mayor complejidad para su puesta en acción. Por otro lado, en músicas distantes a este contexto, prima el mantenimiento de la salud por sobre cualquier otro aspecto que, en términos de identidad, será fuertemente condicionado. Sonidos producidos con la voz que atenten contra dicha salud, no serán considerados como parte del proceso de desarrollo vocal. En ese camino, una cuestión relevante que enlaza al modelo tradicional con el modelo de salud vocal, es el estatus que se le atribuye a la técnica vocal en la enseñanza del canto: por un lado, desarrollar y definir los rasgos sonoro-estéticos de la voz cantada y por otro, la configuración anatomofisiológica pertinente para mantener el aparato vocal en condiciones saludables. Podríamos inferir entonces, que estos dos modelos aun con diferencias sustanciales en su hacer y concebir la actividad, utilizan la técnica vocal como rasgo privilegiado y predominante en la enseñanza del canto.

Siguiendo con las metodologías que subyacen en paralelo con la rápida emergencia musical y el desarrollo tecnológico del siglo XIX, podemos identificar un interés particular por aspectos descuidados en los anteriores modelos en cuanto al análisis de la producción vocal para el canto. Aspectos contextuales, psicológicos, corporales y emocionales que participan en el complejo accionar de la voz en un cantante, se ponen en evidencia ante un mundo en el que las exigencias tanto técnico-musicales y estéticas, así como performativas se convierten en el centro de atención y desarrollo. Ante estos requerimientos, aparece un modelo que aquí hemos denominado "de experiencia corporal" que intenta atender a tales aspectos a través de ejercicios relacionados con la configuración muscular, respiratoria y corporal, en una búsqueda por la reconfiguración kinestésica en función del canto. Las artes escénicas y performativas se integran en la composición de esta línea. Algunas propuestas que pueden relacionarse con esta 
perspectiva teórica, conceptual y metodológica, basada principalmente en la experimentación del cantante, son la Educación funcional de la voz (Eugene Rabine, 2011), Singing for the Stars (Seth Riggs, 1992), Teoría y técnica de la voz (Laura Neira, 2009) entre otros. Por otro lado, El lenguaje del cuerpo (Alexander Lowen, 1997), Autoconciencia del movimiento (Moshe Feldenkrais, 1985), El entrenamiento autógeno (Johannes Heinrich Schultz, 1969), entre otros, muy vinculados con el tratamiento corporal.

Las diferencias observables en estas propuestas son relativamente sustanciales. Por ejemplo, Rabine desarrolla un trabajo de autoconciencia apoyado en la fisiología vocal y la configuración corporal; esta le permite al cantante construir ejercicios suficientes para sus necesidades a partir del reconocimiento de su configuración laríngea y corporal. Por su parte, Seth Riggs atiende al desarrollo vocal desde el nivel del habla y la independencia de algunos músculos no laríngeos que permitirían evitar patologías, y Laura Neira, realiza una transposición de actividades musculares de hombros, cuello y cabeza hacia la parte costodiafragmático-pelviana, con el fin de una sensación placentera en el cuerpo y en la emisión.

Estos métodos comparten dos rasgos comunes; por un lado, en todos hay una mirada que cuestiona y eleva el lugar que ocupa el cuerpo en la acción vocal, el aparato vocal como instrumento interoceptivo y el impacto de los hábitos que termina constituyendo la concreción de un determinado sonido. El segundo rasgo tiene que ver con el interés por conocer y hacer consiente la experiencia corporal que tiene cada cantante en su contexto de desenvolvimiento, con miras a un uso de la voz en el canto desde la organicidad del hacer diario.

Esta línea plantea el canto como una actividad en la cual el sonido no es dependiente de la técnica vocal o la ejercitación del aparato fonador, sino que requiere de un análisis perceptual y propioceptivo consiente para la consecución de una actividad totalmente orgánica en las relaciones humanas como lo es la voz en su rasgo base de oralidad. Para este modelo, la concepción de sonido tiene que ver con la posibilidad de producir la voz con una total distensión fisiológica y corporal, siempre partiendo de una base fundamentalmente hablada. La resultante a la que aspiran desde esta mirada, es un sonido propio, orgánico y por sobretodo, identitario. El cantante entonces, se relaciona con su memoria kinésica, basada en un contexto experiencial que le ha significado tensiones y usos de su cuerpo -en su gran mayoría- innecesarios para esta práctica. La metodología de este modelo se asienta en el uso de una variada serie de ejercicios físicovocales, un proceso de concientización corporal y el habla como modo de 
producción primario. Los profesionales dedicados a la voz que adscriben a este modelo, han transitado un camino experiencial en el cual vieron reflejado lo complejo de esta actividad cuando se desvincula a la voz de la totalidad del cuerpo o se la centra exclusivamente en un aspecto técnico.

Respecto al repertorio en esta perspectiva, las músicas de tradición clásica no son una base de la cual se parta para producir música. De alguna manera, el repertorio que escoge el cantante no es el centro de atención -siempre que este no promueva el deterioro vocal-, sino más bien, que su accionar sobre este, se encuentre acorde al planteamiento del modelo. En lo que respecta al desarrollo vocal en términos de flexibilidad y eficacia, los límites del modelo se encuentran en las exigencias de algunos repertorios, incluido el canto lírico/coral. En otras palabras, su enfoque, fundamentalmente ligado al habla y el tratamiento distendido del cuerpo, deja un vacío frente a las exigencias que demandan algunos estilos en lo que concierne, por ejemplo, a extensión o intensidad. Así entonces, si un cantante tiene un rango vocal acotado debido al tipo de música que interpreta, estos métodos obraran sobre esa voz sin buscar ampliarlo. La enseñanza desde este lugar pone en discusión algunos supuestos de la voz en el canto y acentúa la diferenciación entre las músicas académicas-clásicas o cultas, a las que se les atribuye una súper exigencia, en contrapartida con las músicas populares de baja complejidad (Middleton, 1990) y producción vocal más cercana al habla (Cobeta, Nuñéz y Fernández, 2013; Beltramone y Shifres, 2016), en gran medida por la utilización de tecnologías que modifican o amplifican el sonido como el micrófono.

En este sentido cabe preguntarnos, independientemente del modelo al que se adscriba o considere apropiado, ¿cuáles son las posibilidades que la voz humana puede tener sin pensar en su anclaje a repertorios, estilos o estéticas? Y ¿qué metodologías podemos utilizar y proponer para poder conocer dichas posibilidades de la voz, esto sin que haya una predominancia e idealización de un sonido especifico ligado a un tipo de canon o estereotipo?

\section{Posicionamiento de la laringe: una perspectiva metodológica próxima a las músicas populares}

Para comprender el posicionamiento laríngeo y su vinculación con el sonido es necesario describir algunas cuestiones de orden fisiológico que nos permitirán dialogar con la propuesta que se presenta. Entonces, al emitir un sonido vocal, una cadena compleja de movimientos musculares y 
cartilaginosos se pone en acción. Uno de estos movimientos es el que realiza verticalmente la laringe durante la producción de sonido. Si bien, no es posible palparla en su totalidad de forma externa, hay una protuberancia cartilaginosa en el cuello, comúnmente denominada nuez o manzana de Adán, que puede ayudarnos a comprender el movimiento de este órgano y su relación con los sonidos que se producen.

El movimiento laríngeo se desarrolla en consonancia con las particularidades que tiene el canto. El sostén de un sonido en el tiempo, el movimiento ascendente y descendente, así como saltos interválicos de poca o gran extensión son algunas de las tareas que debe sortear la laringe y que suponen, movimientos abruptos o delicados. El camino metodológico tradicional para responder a esto ha sido el descenso de la laringe, que contribuye al control de presiones aéreas requeridas para la emisión. Los maestros de canto italiano acuñaron a este efecto apoggio -en principiopara hacer referencia a un mecanismo de control de aire a partir de los músculos abdominales (Lyons y Stevenson, 1990). El término se extendió para hablar de la laringe debido a que, la presión que se ejerce para controlar el aire espiratorio, generaba un descenso de la misma y, por ende, un apoggio para sostenerse. En esa línea, el descenso contribuiría con la aparición del formante ${ }^{2}$ del cantante, un aspecto acústico relevante para algunos maestros y cantantes. Del mismo modo, este descenso laríngeo suele considerarse un objetivo para la pedagogía y metodología de corte tradicional al brindar estabilidad, seguridad, dominio en la emisión y sustentabilidad a futuro (Perelló et al., 1982; Sundberg, 1979).

Así como en las músicas pertenecientes al repertorio académico se desarrolla un modo de producción vocal que, estaría próximo al mayor descenso posible de la laringe, en los modos de producción vocal de las músicas populares, este movimiento tiende al ascenso parcial o total para producir determinados sonidos, así como parte de una configuración corporal que participa activamente, tanto para la resultante sonora como para la performance implicada en la práctica. De esta manera, los rasgos de movilidad y apoggio que posee la laringe, nos permiten plantear la posibilidad de modificar consiente e inconscientemente su posicionamiento vertical. Así, sería posible descender la laringe, apoyarla y sostenerla en una base en diferentes niveles de su espacio fisiológico, de acuerdo con las características performativas del cantante y el estilo musical, concientizando

\footnotetext{
${ }^{2}$ Concentración de energía acústica que se produce en determinada frecuencia. La aparición de varias concentraciones de energía, así como la relación de estas se conoce como formante del cantante. Esto le proporcionaría al cantante un timbre con una fuerte proyección.
} 
además la presión aérea y laríngea en distintos modos de producción. Esta propuesta metodológica es parte de un trabajo teórico titulado Posicionamiento de la laringe en la voz cantada (Machuca y Pérez, 2018), en el que se describen algunos aspectos fisiológicos, perceptuales y sonoros de las posiciones laríngeas. En dicho trabajo, se revisaron estudios de investigación relacionados con el movimiento vertical de la laringe, así como la relación que dicho movimiento tendría con la práctica cantada $\mathrm{y}$ algunos estilos musicales. A su vez, se presentaron y describieron tres niveles generales en los cuales puede pensarse la posición y el apoggio laríngeo (figura 1), (i) Nivel profundo, (ii) Nivel intermedio, y (iii) Nivel elevado. A continuación, haremos una síntesis de estos niveles.

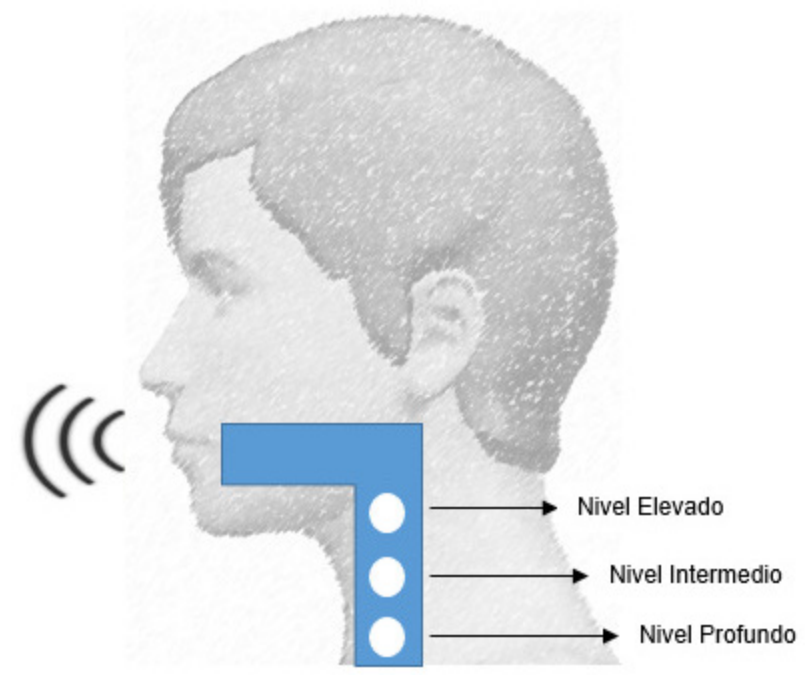

Figura 1. Esquema ilustrativo de los niveles laríngeos.

El primer nivel denominado profundo, se caracteriza fisiológicamente por la apertura y redondez del tracto vocal (cavidad oral, nasal, faríngea y laríngea), cuello ensanchado, fuerte presión aérea costo-abdominal, así como una alta cantidad de energía y exigencia corporal. Su sonido, está definido por intensidades significativamente altas que contribuyen al despliegue de armónicos y su agrupamiento en formantes. Esto último es de suma importancia en lo que respecta a la resonancia de la voz en el cuerpo y en el espacio acústico. La extensión vocal está generalmente ceñida al concepto de tesitura, aunque no es condición sine qua non. El nivel profundo en su descenso laríngeo más pronunciado, se relaciona principalmente con el canto lirico, coral y operístico, no obstante, en un estado medio podemos vincularlo con estilos como el bolero, tango y folclore de principios del S. XX.

En el nivel intermedio, nos encontramos con un estrechamiento del tracto vocal y acortamiento de la cavidad faríngea. Esto se debe al cambio en la 
configuración general de la producción sonora, lo que conlleva una disminución de la presión aérea y laríngea propia del nivel anterior. A su vez, los aspectos corporales que se desprenden en este nivel deben equilibrarse de tal modo que eviten posibles tensiones o constricciones. La cantidad de energía que se requiere es significativamente inferior al nivel profundo, al igual que el involucramiento corporal en términos de fuerza. Este modo de producción vocal podríamos asociarlo con estilos como el rock, jazz, pop y balada entre otros, dado que su timbre -perceptualmente-tiende a la brillantez. Una de los rasgos diferenciados y que cobra relevancia con respecto al nivel profundo, es el uso de la tesitura; aquí, no se presenta en su sentido más conservador, por el contrario, el uso de la total extensión vocal -en la que se incluyen los registros, fry, pecho, falsete, cabeza, silbido-, se convierte en un objetivo enteramente relacionado con la estética y los estilos musicales.

El nivel elevado nos trae una disminución de la presión aérea y por ende una presión laríngea sumamente baja. Esto permite que la laringe se apoye en un lugar correspondiente a dicha cantidad de presión, permitiendo emitir sonidos sin problema. Corporalmente nos encontraremos con un cuello delgado, una apertura mínima del tracto vocal y, un gasto energético mínimo del mismo modo que la intensidad corporal. La suavidad y calidez tímbrica son el fuerte de este nivel, así como la flexibilidad al recorrer toda la extensión vocal y pasar de un registro a otro - passaggio- sin mayor notoriedad. Se lo puede identificar particularmente con el bossa nova y el jazz contemporáneo, no obstante, este nivel suele utilizarse en paralelo con el intermedio, siendo esta una combinación que aparece con frecuencia en la interpretación de músicas populares. A modo de ejemplificación, presentamos tres casos en los que podemos identificar algunas de las características mencionadas para cada nivel laríngeo.

El nivel profundo podemos reconocerlo en la interpretación del aria Nessun Dorma (tercer acto de la ópera Turandot de Giacomo Puccini) realizada por el tenor alemán Jonas Kaufmann en un ensayo (Kaufmann, 2015). El nivel intermedio lo encontramos en la voz del cantautor y compositor cubano Amaury Gutiérrez y el cantante español David Bisbal, al interpretar Dime corazón (Bisbal y Gutiérrez, 2011).

Por último, el nivel elevado puede ser escuchado en la canción "Wave" del cantautor, compositor y pianista brasileño Tom Jobim, en las voces de su hija y su nieto, María Luiza Jobim y Daniel Jobim (Jobim y Jobim, 2009), así como en las voces de los cantautores chilenos Mon Laferte y Manuel García, en el tema “La danza de las libélulas” (García y Laferte, 2020). 
Una de las grandes problemáticas que se plantean en el canto es sobre el estatus que se le confiere a la voz siendo esta parte de nuestro cuerpo. Por ello, la experiencia corporeizada del acto performático es sin duda un aspecto que no aparece cuando pensamos en la enseñanza-aprendizaje del canto. Las líneas de trabajo enfocadas en la voz desde aspectos fisiológicos tendientes a la salud vocal o, aquellas asociadas a una perspectiva corporal de distensión total, desdibujan muchas veces el escenario musical en el cual se desenvuelve el cantante, esto sin mencionar los aspectos emocionales y afectivos que se ven involucrados.

Sin duda, la fisiología y la distensión corporal en la producción vocal es de suma importancia si deseamos cierta sustentabilidad de nuestra voz a largo plazo. No obstante, el desarrollo de la voz cantada implica primeramente una reflexión en torno a las formas sonoro-expresivas que se generan en su entorno natural y social. Aquellas particularidades que, a lo largo de nuestras interacciones socioculturales se van constituyendo como experiencias corporeizadas, materializadas en el modo expresivo sonoro que emerge de nosotros. En esa perspectiva, el desarrollo vocal no está condicionado a las características específicas de un estilo o a una estética que define el cómo de la resultante sonora, sino que comprende la voz como un cuerpo sonoro-expresivo inherentemente humano y social, que se desenvuelve fundamentalmente en sus posibilidades expresivas y que, ante las diferentes prácticas musicales, conjuga la singularidad del cantante y el fenómeno musical en una performance que emerge con cada actuación.

Los ejemplos musicales presentados, además de representar las características mencionadas de los niveles laríngeos, también permiten identificar aspectos expresivos que inciden inevitablemente en la voz cantada. En cada ejemplo, la intencionalidad de las frases, la energía puesta en ellas, su corporeidad, y aún más importante, la vinculación que se establece entre la performance del cantante y los oyentes, hacen posible reconocer esos rasgos expresivos. Independientemente del desarrollo físico que demanda un estilo musical u otro, las formas expresivo-sonoras trascienden la ejercitación y control fisiológico para ser parte de un complejo performático que involucra la experiencia y el modo en el que significamos la música. Las posiciones laríngeas contribuyen a este propósito en la medida que son entendidas como parte de una práctica musical corporeizada, su desarrollo debe estar relacionado con las formas expresivas que proponen las diferentes músicas, así como con las experiencias que tenemos con ellas. La heterogeneidad, propia de las músicas populares, es una complejidad que puede abordarse con la variabilidad que pueden proporcionar las posiciones laríngeas. 
En ese camino, la propuesta metodológica que aquí presentamos, constituye un desarrollo de las ideas de la investigadora y maestra de canto estadounidense conocida como Jo Estill en el método Estill voice training. Según ella, todos tenemos la capacidad de cantar de diferentes maneras con solo cambiar la configuración del tracto vocal, o sea, la cavidad oral, nasal, faringe y laringe. Este método, plantea los movimientos de la laringe como parte de la versatilidad estilística, definiendo así una laringe alta, media y baja. Su programa se basa en la ejercitación y control de "trece figuras vocales": control de cuerdas vocales, cuerdas falsas, grosor de las cuerdas, cartílago tiroideo, cartílago cricoides, laringe, velo del paladar, lengua, esfínter ariepiglótico, mandíbula, labios, cabeza-cuello y torso (McDonald, Obert y Steinhauer, 2005). Su trabajo se vincula posteriormente con el Belting y Twang en tanto cualidades vocales que se desarrollan a lo largo del programa.

Como mencionamos anteriormente, la experiencia corporeizada en la cual se involucra el entorno natural y social, así como las grandes posibilidades expresivas de la voz humana, no suele aparecer en el marco de metodologías de corte tradicional, salud vocal o corporales. En ese camino, la propuesta de Estill es de sumo interés en tanto demuestra que las posiciones de la laringe inciden en la resultante sonora y pueden ser utilizadas en función de determinados estilos musicales, sin embargo, se orienta al dominio de los aspectos fisiológicos que configuran la producción vocal. Propone una línea de trabajo anclado a la idea mecanicista en la que la ejercitación y dominio del artefacto vocal, aseguran un abordaje de diversos estilos musicales, dejando en segundo plano todo el complejo experiencial corporal, psíquico y fenomenológico que se encuentra involucrado en la performance cantada.

Ahora bien, una metodología que aborde todas las músicas en las que el canto se encuentra presente es bastante compleja, teniendo en cuenta los contextos y performatividades en las que se inserta cada práctica y con las cuales, escasamente tenemos contacto. Sin embargo, creemos que un trabajo que nos aproxime lo más posible a otros modos de producción vocal, es un lugar óptimo de partida para aquellos aprendices que buscan un modo de cantar fuera de los lineamientos estrictamente académicos en los que se desarrolla la voz o simplemente para aquellos que su práctica musical se orienta hacia estéticas diferentes.

En este sentido, la propuesta que se presenta yace en un marco pedagógico que considera a la voz cantada como un cuerpo expresivo, emergente de las experiencias y significaciones de nuestro mundo individual y social, 
así como de la comprensión de los diversos escenarios performáticos de la práctica musical. Las metodologías serán entonces herramientas que contribuyan al desarrollo de la voz sin condicionar aquellas formas excluidas por atentar contra la salud vocal, sin dejar de lado su importancia, pero repensando su utilización de modo que, manteniendo la salud, permitan producciones vocales diversas. Nos referimos entonces, a un desarrollo que involucra la concientización de movimientos internos y externos, así como al cuerpo sentiente como parte de la exploración de formas en que el sonido y los movimientos hacen eco en el cuerpo-mente, comprendido dentro de un proceso de experiencia a priori y a posteriori, corporeizado, intersubjetivo, multimodal y cultural (Martínez, 2014).

\section{Consideraciones finales}

Es interesante observar el gran avance que ha tenido el canto a lo largo del tiempo con la innumerable cantidad de estilos musicales que podemos encontrar a diario a través de los medios de comunicación digital. No podemos decir esto sin mencionar todas aquellas músicas que, en el silencio de paradigmas predominantes, también evolucionaron y constituyeron modos de producción vocal como parte de su identidad. Es claro que los modelos de enseñanza y con ellos sus metodologías, han compuesto un corpus direccionado a supuestos y concepciones ontológicas particulares de la voz y el canto. Los modelos que se describieron dan cuenta, aun con sus diferencias sustanciales, que hay una emisión sonoro-vocal que se considera apropiada, especifica y relacionada al modo de producción y la estética musical académica. ¿Qué ocurre entonces con los modos de producción vocal que se alejan de tal emisión?

En los últimos años, diversos profesionales han realizado aportes al tema. Tal es el caso del musicólogo y profesor de teoría musical Walter Everett (2008), quien se interesó por la producción vocal en el rock, proporcionando descripciones de los procedimientos que desarrollan los cantantes en relación a la conducción vocal, tesituras, registros o tipos de articulación. Del mismo modo el musicólogo Allan Moore (2012) realizó, entre otros, trabajos asociados con la interpretación y significación de la canción popular grabada, haciendo análisis de los recursos expresivos que fueron utilizados en relación con las poéticas y posibilidades de las producciones grabadas.

No obstante, el abordaje de las músicas populares en el escenario educativo institucional parece continuar ligado a la trasposición directa e indirecta 
de formas de la práctica musical académica sin la comprensión de los contextos de emergencia de dichas músicas, así como de los significados de sus formas de expresión sonoro-vocal. Pensamos que la heterogeneidad musical, así como el complejo y basto universo respecto a los modos de producción vocal, hacen necesaria la investigación, análisis, comprensión y reflexión de la voz cantada en el pensamiento pedagógico, dando paso al desarrollo y adaptación de metodologías próximas a las performances cantadas de las músicas populares.

Independientemente de la salubridad y sustentabilidad que pueda ofrecer un modelo de canto, recordemos que los sonidos, tímbricas, emisiones y maneras en las que producimos la voz, son un rasgo expresivo básico de lo humano. Esto debiera ser lo suficientemente importante como para construir metodologías sustentables que repliquen dichas formas expresivas, en un corpus musical posible de ser incorporado al ámbito educativo. Los escenarios en los que se han producidos grandes avances con respecto a la compresión de la voz, también entienden los aspectos performativos como parte inherente de su práctica. El claro ejemplo lo tenemos con el canto lírico/coral, un espacio de basta historia que ofrece gran cantidad de posibilidades metodológicas. Sin embargo, asumir que la resultante sonora que subyace en ese contexto, es el fundamento para la producción de otras formas vocales, es vulnerar las prácticas y producciones de otros espacios, así como aminorar el acervo musical de una cultura, tal como se evidencia en los diversos trabajos mencionados anteriormente. En palabras de Nicholas Cook "hemos heredado del pasado un modo de pensar en la música que no puede hacer justicia a la diversidad de prácticas y experiencias que esa pequeña palabra, 'música', expresa en el mundo actual" (2001, p. 29).

El contexto latinoamericano evidencia una mirada alejada de su propio espacio para dar con maneras de producción y enseñanza generalizada, "las fragmentadas narraciones estéticas, musicológicas, etnomusicológicas y folklorológicas, prolongan sus constructos sobre métodos y enfoques diversos que continúan emanando de los centros hegemónicos de Europa y los Estados Unidos" (Hernández Baguer, 2011, p. 66). Basta realizar una simple comparación entre los espacios de formación general, artística y musical latinoamericano, con los espacios europeos y norteamericanos. La diferenciación es tal, que adoptar un modelo sin considerar los contextos en los que se desarrolló y puso en práctica, sería caer en generalizaciones y reduccionismos. La voz es un aspecto expresivo primigenio tan significativo en tanto atributo sonoro comunicacional y corporal, que define nuestra entidad e identidad individual y 
colectiva. Las dimensiones que la componen son, en suma, el panorama pedagógico y metodológico a considerar en cuanto al canto se refiere.

Finalmente, consideramos desde una perspectiva de enseñanza-aprendizaje centrada en el alumno como un sujeto crítico-reflexivo que trae consigo experiencias y conocimientos, así como diferentes corporeidades y modos de significación, que este tipo de abordaje podría ser útil siempre que se haga una revisión ontológica de la voz y el canto en el contexto latinoamericano, entendiendo que esta hace parte de las complejas y grandiosas posibilidades humanas, desprovista de delimitaciones de orden cultural, estético, estilístico o de género. Si bien no podemos negar la tradición, consideramos que la configuración de metodologías pedagógicas debe comprender y dialogar con los contextos en los que cada práctica musical tiene lugar. 


\section{Bibliografía}

» Augustin, K. (2012). Os Castrati: visão holística da prática da castração na música. Música e Linguagem - Revista do Curso de Música da Universidade Federal do Espírito Santo, 1(1), 68-82.

»Barriga, M. (2006). La educación musical durante la Colonia en los virreinatos de Nueva Granada, Nueva España y Río de la Plata. El Artista, 3, 6-23.

»Beltramone, M. y Shifres, F. (2016). La incidencia de los modelos de canto lírico y popular en el desarrollo de las habilidades musicales. En Actas del Primer Congreso Internacional de Música Popular. Epistemología, Didáctica y Producción. La Plata: Universidad Nacional de La Plata, Facultad de Bellas Artes. Recuperado de http://congresomusicapopular.com.ar/images/Actasy-Memorias-1er-Congreso-de-Musica-Popular.pdf

» Cobeta, I., Núñez, F. y Fernández, S. (2013). Patología de la voz. Barcelona: Marge Médica Books.

" Cook, N. (2001). De Madona al canto gregoriano. Una muy breve introducción a la música (Traductor Gago, L.). Madrid: Alianza Editorial.

» Everett, W. (2008). Foundations of Rock: from 'Blue Suede Shoes' to 'Suite: Judy Blue Eyes.' Nueva York: Oxford University Press, Inc.

» Feldenkrais, M. (1985). Autoconciencia a través del movimiento. Barcelona: Paidós.

» García, M. (1840). Tratado completo del arte del canto. Buenos Aires: Ricordi Americana.

" Guerrero, H. (2015). La técnica vocal en la Edad Media. Diez siglos de práctica del canto. (tesis doctoral). Universidad Rey Juan Carlo, Madrid, España.

» Hemsy de Gainza, V. (2002). Pedagogía vocal: Dos décadas de pensamiento y acción educativa. Buenos Aires: Lumen.

»Hernández Baguer, G. (2011). Latinoamérica en el imaginario de Argeliers León. Para una interpretación de su cancionero cubano. Boletín música. Revista de música latinoamericana y caribeña, 28, 63-81.

»Husson, R. (1965). El canto (Traductora Ling D.). Buenos Aires: EUDEBA.

» Kingsbury, H. (1988). Music, Talent and Performance: A Conservatory Cultural System. Philadelphia: Temple University Press.

» Le Huche, F. y Allali, A.. (2004). La voz: anatomía y fisiología de los órganos de la voz y del habla. Barcelona: Masson.

» Lopes, E., Basso, L. y de Andrade, M. (2015). Postura corporal, voz e autoimagem em cantores líricos. Revista Academica de Musica, 31, 74-85. doi: 10.1590/permusi2015a3104

» López, I. y Vargas, G. (2010). La música popular y el modelo hegemónico de 
enseñanza instrumental. Actas IX Reunión anual de SACCoM. Recuperado de http://www.saccom.org.ar/ACTAS_CONGRESOS/9na_SACCoM.pdf

» Lowen, A. (1997). El lenguaje del cuerpo: dinámica física de la estructura del carácter. Barcelona: Herder Editorial.

» Lyons, J. y Stevenson, L. (1990). POPS: Principles of Pop Singing. New York: Schirmer Books.

» Machuca, D. y Pérez, J. (2018). Posicionamiento laríngeo en la voz cantada: repensando los modos de producción vocal en la música popular y su abordaje en la pedagogía vocal. En S. Romé (Coord.). Segundo Congreso Internacional de Música Popular. Epistemología, Didáctica y Producción. Congreso llevado a cabo en Facultad de Bellas Artes, UNLP, La Plata.

» Machuca, D., Valles, M. y Pérez, J. (2019). Pedagogías de canto en la ciudad de la plata: análisis de la experiencia docente en un grupo de profesores de canto. En Actas del $7^{\circ}$ Congreso Latinoamericano en Formación Académica en Música Popular. Sec. Investigación y Extensión del IAPCH-IAPCH-UNVM. Recuperado de https://drive.google.com/file/d/1HaN1V8vLX18EXIdjOQpD zP9qoiAa7LhQ/view

» Martínez, I. C. (2014). La base corporeizada del significado musical. En S. Español (Comp.). Psicología de la música y del desarrollo: una exploración interdisciplinaria sobre la musicalidad humana (pp. 71-110). Buenos AiresBarcelona-México: Paidós.

» McDonald, M., Obert, K. y Steinhauer, K. (2005). Estill Voice Training System: Compulsory Figures for Voice Control. Level One. Pittsburgh, Pa: Estill Voice Training System International.

» Middleton, R. (1990). Studying Popular Music. Philadelphia: Open University Press.

» Moore, A. (2012). Song Means: Analysing and Interpreting Recorded Popular Song. Farnham: Ashgate Publishing Limited.

» Musumeci, O. (2002). Hacia una educación de conservatorio humanamente compatible. Actas de la Segunda Reunión Anual de SACCoM. Recuperado de http://www.saccom.org.ar/2002_reunion2/SesionesTematicas/Musumeci. htm

»Neira, L. (2009). Teoría y técnica de la voz: el método Neira de educación vocal. Buenos Aires: Akadia Editorial.

»Peckham, A. (2000). The Contemporary Singer: Elements of Vocal Technique. Boston: Berklee Press.

»Peckham, A. (2006). Vocal Workouts for the Contemporary Singer Boston: Berklee Press.

»Perelló, J., Caballé, M. y Guitart, E. (1982). Canto - Dicción (foniatría estética). Barcelona: Editorial Científico-Médica.

» Rabine, E. (2011). Educación funcional de la voz. Método Rabine. Buenos Aires: Editorial Dunken. 
" Riggs, S. (1992). Singing for the Stars: A Complete Program for Training your Voice. USA: Alfred Publishing.

»Sánchez, N., Guimarães, A. y de Andrade, M. (2017). Popular and Classical Female Singers: Acoustic Comparison of Voice Use in the Song Melodia Sentimental (Sentimental Melody) by Heitor Villa-Lobos. Journal of Voice, 33(2), 255.e27-255.e37.

»Schultz, J. (1969) El entrenamiento autógeno. Barcelona: Editorial CientíficoMédica.

"Shaw, K. (1987). Vocal Jazz Style. Milwaukee. WI: Hal Leonard.

"Spivey, N. (2005). Popular Song and Music Theater: Teaching Music Theater Singing: One Teacher's Journey. Journal of Singing, 62(2), 199-202.

»Spivey, N. (2008). Popular Song and Music Theater: Music Theater Singing... Let's Talk. Part One. On the Relationship of Speech and Singing. Journal of Singing, 64(4), 483-489.

»Stein, H. (2000). El arte de cantar: su dimensión cultural y pedagógica. Revista musical chilena, 54(194), 41-48.

»Sundberg, J. (1979). Perception of Singing. Speech Transmission Laboratory Quarterly Progress and Status Report, 1, 1-48.

» Titze, I. (2010). Fascinations with the Human Voice. Utah: National Center for Voice.

»Zegree, S. (2002). The Complete Guide to Teaching Vocal: Including Pop and Other Show Styles. Dayton, OH: Heritage Music.

\section{Referencias audiovisuales}

» Bisbal, D. y Gutiérrez, A. [Pepa Fernando] (01 de febrero de 2011). David bisbal \& amaury gutierrez dime corazón bg sub [Archivo de video]. Recuperado de https://www.youtube.com/watch?v=Kykq3cLzWws\&list=PL D410D8EF97C3CFDD\&index=11

» García, M. y Laferte, M. [manugarpez] (21 de febrero de 2020). Manuel García \& Mon Laferte - La Danza de las Libélulas [Archivo de video]. Recuperado de https://www.youtube.com/watch?v=sJDG60HiGkw

» Jobim, D. y Jobim, L. [fernnn1] (18 de octubre de 2009). Wave - Daniel \& Luiza Jobim +Lyrics [Archivo de video]. Recuperado de https://www. youtube.com/watch?v=ieGC1CPMHc8

» Kaufmann, J. [JonasKaufmannVEVO]. (02 de noviembre de 2015). Jonas Kaufmann - Turandot, Atto III: "Nessun Dorma" [Archivo de video]. Recuperado de https://www.youtube.com/watch?v=xN-JCdM4or0 


\section{Biografías / Biografias / Biographies}

\section{Daniel Machuca Téllez}

Licenciado en música con orientación en Educación Musical de la Facultad de Bellas Artes (Universidad Nacional de La Plata). Docente adscripto en la catedra Audioperceptiva I y II de dicha facultad. Tesista de posgrado de la Especialización en Lenguajes Artísticos y Doctorando en Artes de la FBA-UNLP y becario de investigación por la UNLP. De 2015 a 2017 participó como ayudante en la catedra Educación Vocal dirigida por María Verónica Benassi. Realiza tareas de investigación sobre la voz en el Laboratorio para el estudio de la experiencia musical e integra el proyecto PPID/B010, "Improvisación y oralidad en la música popular. Hacia una definición de los aspectos corporeizados, fenomenológicos y gramaticales de la performance como ontología de la acción", dirigido por Joaquín Blas Pérez. Se ha dedicado a estudiar de manera autodidacta la voz cantada y los modos de producción vocal en los diferentes estilos de la música popular. Desde 2016 desarrolla el proyecto independiente "Espacio de Desarrollo Vocal Para el Canto Canere" en la cual participa como docente.

\section{Mónica Valles}

Profesora de Iniciación musical y Profesora Universitaria de Educación Musical, egresada de la Faculta de Bellas Artes (Universidad Nacional de La Plata). Investigadora Categoría III del Programa de Incentivos SPU Ministerio de Educación de la Nación. Profesora Adjunta de las cátedras Audioperceptiva I y II de la Facultad de Bellas Artes (UNLP). Co-directora del Proyecto Acreditado 11B/298 "La corporeidad de la mente musical. Hacia una definición de su estatura en el estudio de la ontogénesis, la percepción y la performance de la música". Integrante del proyecto "Musicalidad Comunicativa en las Artes Temporales y la Infancia Temprana" PICT 2013-0368 (Agencia Nacional de Promoción de la Ciencia y la Tecnología). Miembro del Consejo Asesor del Laboratorio para el Estudio de la Experiencia Musical (LEEM) de la Facultad de Bellas Artes (UNLP). Miembro del Órgano de fiscalización de la Sociedad Argentina para las Ciencias Cognitivas de la Música. Integrante del dúo de música latinoamericana Peña-Valles (piano, voz y arreglos). 